\begin{tabular}{|c|c|c|}
\hline \multirow{3}{*}{$\begin{array}{l}\text { Case Reports in } \\
\text { Gastroenterology }\end{array}$} & \multirow{2}{*}{\multicolumn{2}{|c|}{ Case Rep Gastroenterol 2014;8:346-352 }} \\
\hline & & \\
\hline & $\begin{array}{l}\text { DOI: } 10.1159 / 000369130 \\
\text { Publisned online: November 6, } 2014\end{array}$ & $\begin{array}{l}\text { (c) } 2014 \text { S. Karger AG, Basel } \\
1662-0631 / 14 / 0083-0346 \$ 39.50 / 0 \\
\text { www.karger.com/crg }\end{array}$ \\
\hline & \multicolumn{2}{|c|}{$\begin{array}{l}\text { This is an Open Access article licensed under the terms of the Creative Commons } \\
\text { Attribution-NonCommercial } 3.0 \text { Unported license (CC BY-NC) (www.karger.com/OA } \\
\text { license), applicable to the online version of the article only. Distribution permitted for non } \\
\text { commercial purposes only. }\end{array}$} \\
\hline
\end{tabular}

\title{
Zenker's Diverticulum: Diagnostic Approach and Surgical Management
}

\author{
Carlos M. Nuño-Guzmán ${ }^{a}$ Daniel García-Carrasco ${ }^{a} \quad$ Miguel Haro $^{a}$ \\ José Arróniz-Jáuregui ${ }^{a}$ Jorge L. Corona ${ }^{b}$ Macario Salcido ${ }^{a}$ \\ Departments of a General Surgery and ${ }^{b}$ Radiology, Antiguo Hospital Civil de Guadalajara \\ 'Fray Antonio Alcalde', Guadalajara, Mexico
}

\section{Key Words}

Zenker's diverticulum $\cdot$ Hypopharyngeal diverticulum $\cdot$ Dysphagia

\begin{abstract}
Zenker's diverticulum (ZD), also known as cricopharyngeal, pharyngoesophageal or hypopharyngeal diverticulum, is a rare condition characterized by an acquired outpouching of the mucosal and submucosal layers originating from the pharyngoesophageal junction. This false and pulsion diverticulum occurs dorsally at the pharyngoesophageal wall between the inferior pharyngeal constrictor and the cricopharyngeus muscle. The pathophysiology of ZD involves altered compliance of the cricopharyngeus muscle and raised intrabolus pressure. Decreased compliance of the upper esophageal sphincter and failure to open completely for effective bolus clearance both lead to an increase in the hypopharyngeal pressure gradient. Different open surgical techniques and transoral endoscopic approaches have been described for the management of $Z D$, although there is no consensus about the best option. We report the case of a 61-year-old patient with a 7-year history of dysphagia and odynophagia for solid food, which after 2 months progressed to dysphagia for liquids and after 4 months to regurgitation 2-6 h after meals. The patient experienced a $12-\mathrm{kg}$ weight loss. Diagnosis was established by esophagogram, which showed a diverticulum through the posterior pharyngeal wall, suggestive of a ZD. Esophagogastroduodenoscopy showed a pouch with erythematous mucosa. Under general anesthesia, diverticulectomy and myotomy were performed. After an uneventful recovery and adequate oral intake, the patient remains free of symptoms at 4 months of follow-up.

(c) 2014 S. Karger AG, Basel
\end{abstract}

Carlos M. Nuño-Guzmán, MD, MSc

Antiguo Hospital Civil de Guadalajara 'Fray Antonio Alcalde'

Calle 68 No. 138 , Sector Reforma

C.P. 44800, Guadalajara, Jalisco (Mexico)

E-Mail carlosnunoguzman@hotmail.com 
Nuño-Guzmán et al.: Zenker's Diverticulum: Diagnostic Approach and Surgical Management

\section{Introduction}

Zenker's diverticulum (ZD), also known as cricopharyngeal, pharyngoesophageal or hypopharyngeal diverticulum, is an acquired outpouching of the mucosal and submucosal layers (false diverticulum) originating from the pharyngoesophageal junction. This pulsion diverticulum occurs dorsally at the pharyngoesophageal wall between the inferior pharyngeal constrictor and the cricopharyngeus muscle, a region of relative weakness known as Killian's triangle or Killian's dehiscence [1]. ZD is a rare condition with a reported prevalence of $0.01-0.11 \%$ in the general population [2].

ZD may cause dysphagia by two mechanisms: incomplete opening of the upper esophageal sphincter (UES) and extrinsic compression of the cervical esophagus by the diverticulum itself [3]. The pathophysiology of ZD involves altered compliance of the cricopharyngeus muscle and raised intrabolus pressure. Decreased compliance of the UES and failure to open completely for effective bolus clearance both lead to an increase in the hypopharyngeal pressure gradient. Greater intrabolus pressures have been documented in patients with ZD compared with an age-matched healthy population $[4,5]$. Since these diverticula occur in the elderly, UES dysfunction and muscular weakness have been considered [6]. Different open surgical techniques and transoral endoscopic approaches have been described for the management of ZD, although there is no consensus about the best option.

We report a case of ZD in an adult patient, the diagnostic approach and the surgical management.

\section{Case Report}

A 61-year-old male patient was referred to our Department of General Surgery with a 7-year history of dysphagia and odynophagia for solid food, which after 2 months progressed to dysphagia for liquids and after 4 months to regurgitation 2-6 h after meals. Due to fear to eat, the patient had experienced a 12 - $\mathrm{kg}$ weight loss, which represented $15.3 \%$ of his previous total weight $(78.5 \mathrm{~kg})$. During the last 2 months he had suffered intermittent periods of cough, hyaline sputum and nocturnal fever $\left(38^{\circ} \mathrm{C}\right)$.

At presentation, physical examination only showed signs of malnutrition, but no evidence of respiratory tract infection was found. Laboratory blood tests were unremarkable. Esophagogram showed a diverticulum through the posterior pharyngeal wall suggestive of ZD (fig. 1). Esophagogastroduodenoscopy showed a pouch with erythematous mucosa. The patient was scheduled for myotomy and diverticulectomy. Under general endotracheal anesthesia, through an incision at the anterior border of the left sternocleidomastoid muscle, the platysma muscle was divided. The sternocleidomastoid muscle was retracted laterally and the omohyoid muscle exposed and divided. Dissection was carried down ventral to the carotid sheath, which was retracted laterally while the thyroid gland and the larynx were retracted medially. Once the diverticulum was identified and dissected between the esophagus and the prevertebral fascia, resection at the base of the pedicle was performed with a linear stapler (fig. 2). A 3-cm myotomy of the cricopharyngeus muscle was performed prior to diverticulum resection. After an uneventful recovery and adequate oral intake, the patient was discharged on the second postoperative day. On histologic examination there was no evidence of malignancy. Four months after the surgery the patient remains free of symptoms. 
Nuño-Guzmán et al.: Zenker's Diverticulum: Diagnostic Approach and Surgical Management

\section{Discussion}

Esophageal diverticula are rare, with a prevalence of $0.06-4 \%$ based on radiologic and endoscopic series [6]. ZD is the most common esophageal diverticula, representing $75 \%$ of them [7]. The overall prevalence of ZD among the general population is reported to be between 0.01 and $0.11 \%$ [2]. ZD is usually diagnosed between the seventh and eighth decades of life and rarely before the age of 40 years [3]. In a recent review in the United States, the mean age of ZD patients was $73.0 \pm 12.3$ years, with most patients aged $\geq 65$ years $(78 \%)$ and a higher proportion of male patients (55\%) [7]. It consists in a typical pulsion diverticulum (false diverticulum) occurring dorsally at the pharyngoesophageal wall, a region of relative weakness known as Killian's triangle or Killian's dehiscence, bounded by the propulsive oblique inferior pharyngeal constrictor muscle and the transverse fibers of the cricopharyngeal muscle (contributing to the UES) [1].

Among symptomatic ZD patients, 80-90\% complain of dysphagia [3]. Typical symptoms also include regurgitation of undigested food, choking, chronic cough and halitosis. The duration of symptoms at presentation may vary from weeks to several years. As the pouch enlarges and dysphagia increases, symptoms become more severe with resultant weight loss. Physical signs of malnutrition can be observed. Cervical borborygmus, particularly in the presence of a palpable lump in the neck, is almost pathognomonic of ZD. Hoarseness, cough and aspiration pneumonia may be present in 30-40\% of patients. A sudden increase in the severity of dysphagia and/or presence of alarm symptoms, such as local pain and hemoptysis or hematemesis, should raise the suspicion of ulceration or squamous cell carcinoma within the pouch $[3,8]$.

Dysphagia may be caused by two mechanisms: incomplete opening of the UES and extrinsic compression of the cervical esophagus by the diverticulum itself [3]. The pathophysiology of ZD involves altered compliance of the cricopharyngeus muscle or raised intrabolus pressure. Decreased compliance of the UES and failure to open completely for effective bolus clearance both lead to an increase in the hypopharyngeal pressure gradient. Greater intrabolus pressures have been observed in patients with ZD compared with an age-matched healthy population $[4,5]$. Since these diverticula occur in the elderly, UES dysfunction and muscular weakness have been considered [6]. A marked increase in fibroadipose tissue replacement of the cricopharyngeus muscle and normal connective tissue, and fiber degeneration, compromising sphincteric elasticity and sphincter opening, support this hypothesis [3].

Histologically the pouch shows a stratified squamous epithelium. The presence of cancer has been reported in $0.3-7 \%$ of ZD cases. The main predisposing factor in carcinoma is thought to be chronic inflammation of the pouch lining over many years, secondary to food retention. Risk factors for malignant transformation are old age, male gender, longstanding history and larger diverticula. Progressive dysphagia, unintentional weight loss, presence of blood in the regurgitated material, regurgitation of a piece of tumor, odynophagia, melena, hematemesis and hemoptysis should raise suspicion of malignancy. Carcinoma in situ or small carcinomas may not be detected radiologically or by endoscopic examination $[6,8]$.

Esophageal barium swallow constitutes the most important diagnostic test. At the level of the sternoclavicular joint, typical outpouching on the dorsal surface of the esophagus is seen, and its size and position can be assessed. Many authors classify ZD by its size, measured in the craniocaudal direction: small (up to $2 \mathrm{~cm}$ ), intermediate $(2-4 \mathrm{~cm})$ and large $(4-6 \mathrm{~cm})[8,9]$. Dynamic continuous fluoroscopy allows monitoring of the swallowing mechanism and may detect a small diverticulum. Evidence of overflow and aspiration can be seen. Evaluation of the lower esophagus, stomach and duodenum may detect abnormalities 
Nuño-Guzmán et al.: Zenker's Diverticulum: Diagnostic Approach and Surgical Management

such as hiatus hernia or reflux esophagitis. A non-progressive filling defect or loss of the smooth contour of the interior of the pouch should raise suspicion of a carcinoma $[3,8]$. Esophagogastroduodenoscopy is not necessary to confirm the diagnosis of ZD, but it serves to rule out malignancy in the pouch and to exclude other conditions responsible for the patient's symptoms, such as reflux esophagitis or tumors [6, 9].

Since the first successful resection of ZD by Wheeler in 1886 [6], various open surgical techniques have been described for the management of ZD, including diverticulectomy, diverticulopexy, diverticular inversion (all with or without cricopharyngeal myotomy), and cricopharyngeal myotomy alone, although there is no consensus as to the best option $[8,10]$. Mosher in 1917 was the first to treat an esophageal pouch by a transoral endoscopic technique dividing the common septum between the esophagus and the pouch. In 1960, Dohlman and Mattson described the division by coagulation of the septum between the esophagus and the ZD by rigid transoral endoscopy $[8,10]$.

Several factors must be considered when deciding whether to use an open surgical or transoral approach. Clear endoscopic exposure of the diverticulum may be limited in a patient with short neck, decreased hyomental distance, inability to adequately open the mouth, large osteophytes, obesity and redundant mucosa. Physician expertise is of paramount importance $[3,6]$. Insufficient protection or adhesion of the diverticulum sac by the dorsal esophageal wall, and in small diverticula, cutting through this wall to create a common channel will result in perforation. In the event of a failed endoscopic approach, which occurs in $16-68 \%$ of patients, an open surgical procedure is indicated [3].

Surgical repair of ZD is usually performed under general endotracheal anesthesia. An incision is made at the anterior border of the left sternocleidomastoid muscle, with dissection carried down ventral to the carotid sheath until the diverticulum is identified between the esophagus and the cervical spine. The diverticulum is completely dissected, with adequate visualization of its neck $[3,6,9,10]$. The rationale of cricopharyngeal myotomy is based on the assumption that an UES dysfunction is part of the pathophysiology of the disease. The effects will include normalization of the opening size of the UES, reduction of resting UES pressure and decrease in intrabolus pressure. A myotomy is performed approximately $2 \mathrm{~cm}$ proximally into the inferior pharyngeal constrictor to $5 \mathrm{~cm}$ distally through the cricopharyngeal and into the proximal esophagus $[1,3,6,8-10]$.

Following myotomy the ZD may be excised (diverticulectomy), retracted cranially and suspended by suture to the prevertebral fascia or the posterior pharyngeal wall (diverticulopexy), or inverted into the esophageal lumen and oversewn (diverticulum inversion or invagination) $[1,3,6,9,10]$. In case of a small diverticulum $(<2 \mathrm{~cm})$, once the myotomy is performed, the pouch may be left in place. The risks of complications and cancer are small. Most of them will simply vanish after the cricopharyngeal myotomy. Diverticulopexy is also an option $[1,3,6]$. Intermediate-size diverticula can be treated with cricopharyngeal myotomy plus pouch inversion or diverticulopexy [3, 8]. This approach will leave the mucosa intact and the incidence of leakage will decrease, with a shorter hospitalization and earlier oral intake resumption [10]. Potential adverse events of open surgery include fistula formation, abscess, hematoma, recurrent nerve paralysis, difficulties in phonation and Horner syndrome [3]. Larger diverticula may be managed by diverticulectomy or diverticulopexy.

Diverticulopexy of large diverticula may create a bulky mass compressing the pharynx; as such, they should probably be resected. Diverticulectomy was originally followed by manual suture repair. Linear stapling devices allow pouch excision and pharyngeal wall closure in a single step $[3,6]$. Diverticulectomy can be used to manage pouches of any size with the advantage of complete pouch excision, eliminating any risk of pouch mucosa car- 
Nuño-Guzmán et al.: Zenker's Diverticulum: Diagnostic Approach and Surgical Management

cinoma. However, it does carry a higher complication rate including wound infections, hematoma, leakage and fistula formation, surgical emphysema, mediastinitis and recurrent nerve damage. It also requires a longer anesthetic and hospitalization time, making it less appropriate for elderly patients. The use of linear stapler devices has substantially reduced the risk of leak $[3,8,10]$.

Open surgery can effectively resolve symptoms in $90-95 \%$ of patients $[3,10]$. In a recent literature review, an overall morbidity of $10.5 \%$ and a mortality of $0.6 \%$ were observed. When reviewing the trends in the last 20 years, $56.4 \%$ of patients were treated with diverticulectomy plus cricopharyngeal myotomy, which represents the most popular open surgical approach for ZD [10]. Considering the fact that ZD is a disease of the elderly and the possibility of life-threatening complications, minimally invasive transoral endoscopic treatment has gained popularity [10]. In some cases of elderly, medically unfit patients with minimal symptoms, no surgical treatment except careful observation may be indicated [8].

Transoral endoscopic treatment creates a common cavity between the esophagus and the diverticulum. The septum between the two structures contains the cricopharyngeal muscle. With the division of this septum a myotomy automatically is added to the procedure, preventing food accumulation and relieving the outflow obstruction. The endoscopic approach can be accomplished by a rigid or a flexible endoscope. Division of the septum can be performed by electrocautery, $\mathrm{CO}_{2}$ laser, KTP/532 laser, stapler, needle knife or ultrasonic scalpel $[1,3,6,10]$. No substantial differences with regard to symptomatic outcome, length of stay and complications have been found. Some authors indicate endoscopic treatment in high-risk patients while others believe it can be a first-choice therapy [6]. The flexible endoscopic approach is especially useful for patients with poor neck extension and/or limited jaw retraction. This technique can be performed under conscious sedation without general anesthesia and may be most suitable for elderly and comorbid patients $[3,10]$. The majority of studies of endoscopic techniques report a clinical resolution rate of approximately $90 \%$, with recurrence in $10 \%$ [3]. Complications include cervical or mediastinal emphysema, esophageal tear or perforation, dental injury, bleeding, mediastinitis, leaks, respiratory infections, stenosis, recurrent nerve injury, neck abscess, sore throat and gingival laceration $[3,10]$. A recent review found an overall morbidity of $8.7 \%$ and a mortality of $0.2 \%$ [10]. The lack of objective measurements in long-term follow-up is a limitation in most studies. There are no randomized trials comparing techniques. There is no strong evidence to conclude which is the optimal treatment for ZD [10].

The case reported herein is a male patient with typical clinical presentation and with radiographic and endoscopic findings of ZD. Myotomy and diverticulectomy were performed.

\section{Disclosure Statement}

The authors have no conflicts of interest, sponsorship or funding arrangements to declare. 


\section{Case Reports in \\ Gastroenterology}

\begin{tabular}{l|l}
\hline \multicolumn{2}{l}{ Case Rep Gastroenterol 2014;8:346-352 } \\
\hline DOI: 10.1159/000369130 & $\begin{array}{l}\text { C 2014 S. Karger AG, Basel } \\
\text { www.karger.com/crg }\end{array}$ \\
\hline
\end{tabular}

Nuño-Guzmán et al.: Zenker's Diverticulum: Diagnostic Approach and Surgical Management

\section{References}

1 Bizzotto A, Iacopini F, Landi R, Costamagna G: Zenker's diverticulum: exploring treatment options. Acta Otorhinolaryngol Ital 2013;33:219-229.

-2 Watemberg S, Landau O, Avrahami R: Zenker's diverticulum: reappraisal. Am J Gastroenterol 1996;91: 1494-1498.

-3 Law R, Katzka DA, Baron TH: Zenker's diverticulum. Clin Gastroenterol Hepatol 2013, Epub ahead of print.

4 Cook IJ, Gabb M, Panagopulos V, Jamieson GG, Dodds WJ, Dent J, Shearman DJ: Pharyngeal (Zenker's) diverticulum is a disorder of the upper esophageal sphincter opening. Gastroenterology 1992;103: 1229-1235.

5 Peters JH, Mason R: The physiopathological basis for Zenker's diverticulum. Chirurg 1999;70:741-746.

6 Herbella FA, Patti MG: Modern pathophysiology and treatment of esophageal diverticula. Langenbecks Arch Surg 2012;397:29-35.

7 Onwugbufor MT, Obirieze AC, Ortega G, Allen D, Cornwell EE 3rd, Fullum TM: Surgical management of esophageal diverticulum: a review of the Nationwide Inpatient Sample database. J Surg Res 2013;184: $120-125$.

8 Siddiq MA, Sood S, Strachan D: Pharyngeal pouch (Zenker's diverticulum). Postgrad Med J 2001;77:506511.

9 Vogelsang A, Schumacher B, Neuhaus H: Therapy for Zenker's diverticulum. Dtsch Arztebl Int 2008;105: $120-126$.

10 Yuan Y, Zhao YF, Hu Y, Chen LQ: Surgical treatment of Zenker’s diverticulum. Dig Surg 2013;30:207-218.
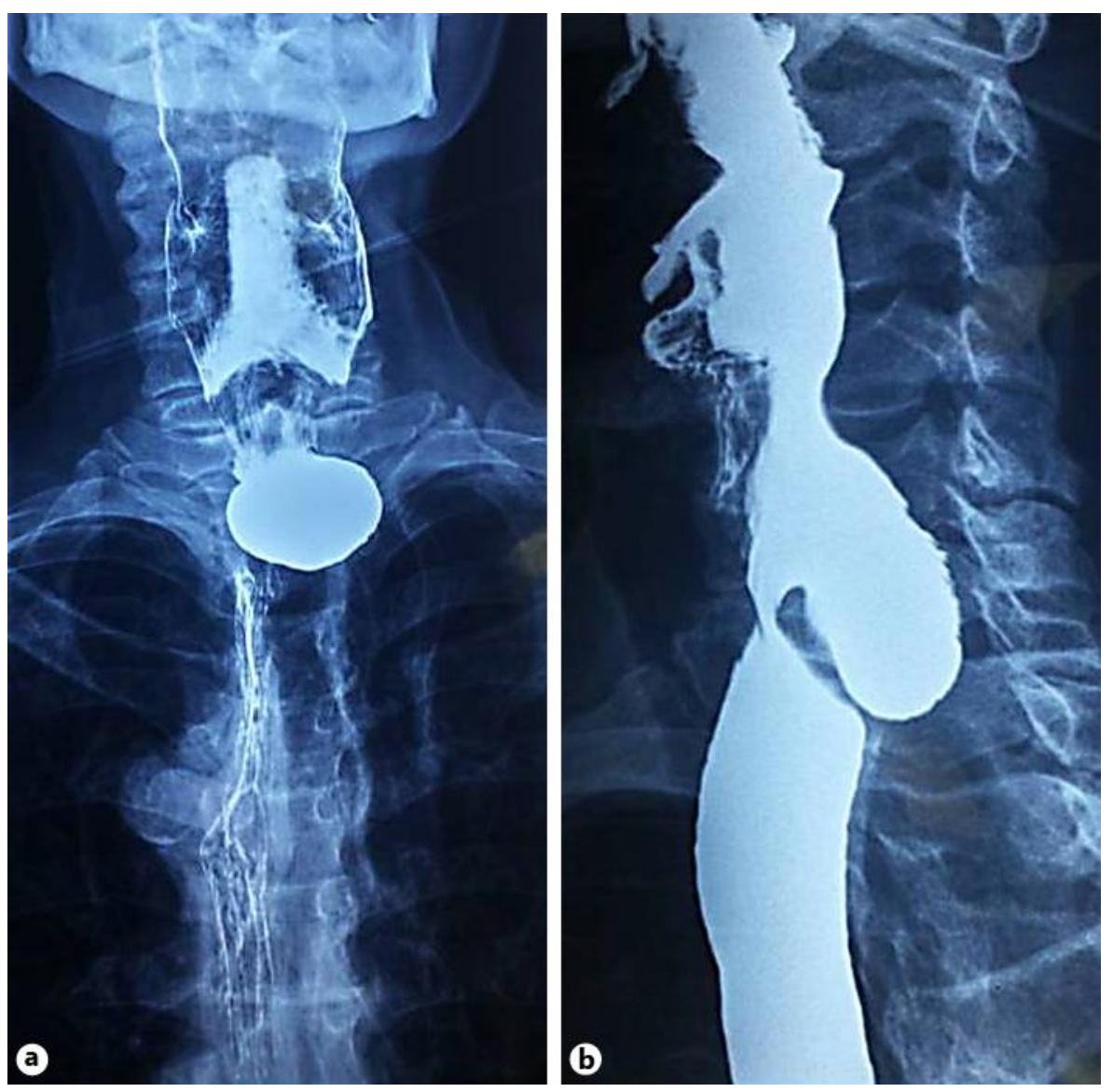

Fig. 1. Esophagogram showing a ZD: anterior-posterior view (a) and lateral view (b). 
Nuño-Guzmán et al.: Zenker's Diverticulum: Diagnostic Approach and Surgical Management
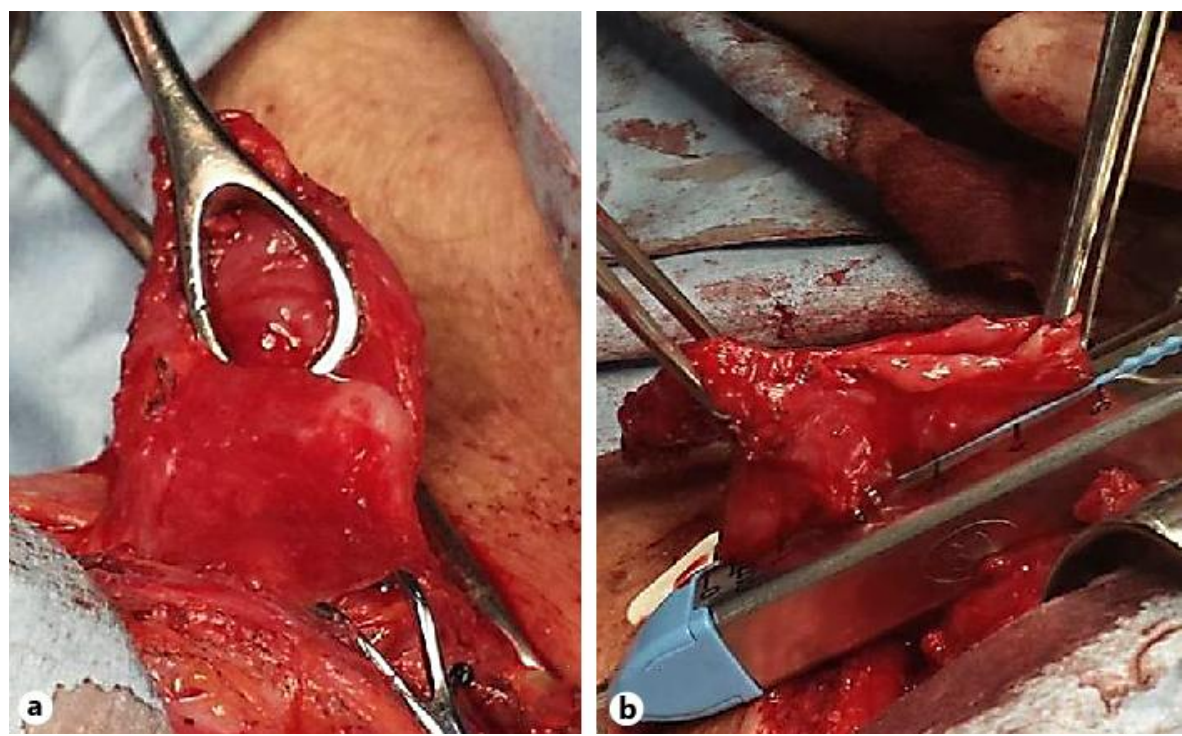

Fig. 2. a Surgical aspect of the ZD. b Resection at the base of the pedicle. 